\title{
Interactive comment on "Uncertainty analysis of hydrological return period estimation, taking the upper Yangtze River as an example" by Hemin Sun et al.
}

\section{Anonymous Referee \#2}

Received and published: 29 March 2017

This paper introduces and describes a quantitative way to assess the uncertainty in estimating return periods by considering different data sampling methods, distribution functions and parameterizations estimation methods. The topic of this manuscript had practical value for the engineering design or the flood risk assessment. In generally, I found this manuscript interesting, technically sound, and well organized. Nevertheless, I think this paper needs some revisions in order to clarify the novelty of the methods. I also suggest the authors to carefully review grammar and spelling throughout the entire manuscript. I have provided some editorial suggestions at the end of this review, but I may have missed some.

The conclusion "SM is the main source of uncertainty for the stations with significant 
trend, while the DF contributed most to the uncertainty for the stations without clear trend" only basin on two hydrological stations. I think this kind of conclusions should be based on statistical results. Therefore, I strongly recommend either presenting a strong argument in favor of only two stations or better using higher-number stations.

Also, the method of DPOT is not explained clearly, I do not understand why the authors choose only the station with significant downward trend, what if the series have a significant increasing trend? Wound the DPOT also be a better sample method to reduce the uncertainty?

The authors should clarify why the Chi-squared method was selected for fit test.

The abbreviations of distribution is inconsistent in the manuscript. Please check and correct.

In the abstract: "But uncertainties. ..." should replace by "though uncertainties".

There lots of mistakes in the superscripts of units in section 2

I suggest the authors provide further practical interpretation of the results presented in the last section.

All the references should be edited according to the format of HESS. The reference listed below was not cited in the manuscript. Kianfar, B., Fatichi, S., Paschalis, A., Maurer, M., and Molnar, P.: Climate change and uncertainty in high-resolution rainfall extremes, Hydrol. Earth Syst. Sci. Discuss., doi:10.5194/hess-2016-536, 2016

Interactive comment on Hydrol. Earth Syst. Sci. Discuss., doi:10.5194/hess-2016-566, 2017. 